\title{
CLASSIFICATION OF INVARIANT CONES IN LIE ALGEBRAS
}

\author{
JOACHIM HILGERT AND KARL H. HOFMANN
}

All Lie algebras in the following are finite dimensional real Lie algebras. A cone in a finite dimensional real vector space is a closed convex subset stable under the scalar multiplication by the set $\mathbf{R}^{+}$of nonnegative real numbers; it is, therefore additively closed and may contain vector subspaces. A cone $W$ in a Lie algebra $\mathfrak{g}$ is called invariant if

$$
e^{\operatorname{ad} x}(W)=W \quad \text { for all } x \in \mathfrak{g} .
$$

We shall describe invariant cones in Lie algebras completely. For simple Lie algebras see [KR82, Ol81, Pa84, and Vi80].

Some observations are simple: If $W$ is an invariant cone in a Lie algebra $\mathfrak{g}$, then the edge $\mathfrak{e}=W \cap-W$ and the span $W-W$ are ideals. Therefore, if one aims for a theory without restriction on the algebra $\mathfrak{g}$ it is no serious loss of generality to assume that $W$ is generating, that is, satisfies $\mathfrak{g}=W-W$. This is tantamount to saying that $W$ has inner points. Also, the homomorphic image $W / \mathfrak{e}$ is an invariant cone with zero edge in the algebra $\mathfrak{g} / \mathfrak{e}$. Therefore, nothing is lost if we assume that $W$ is pointed, that is, has zero edge. Invariant pointed generating cones can for instance be found in $\mathrm{sl}(2, \mathbf{R})$, the oscillator algebra and compact Lie algebras with nontrivial center (see [HH85b, c, HH86a, or HHL87]).

A subalgebra $\mathfrak{h}$ of a Lie algebra $\mathfrak{g}$ is said to be compactly embedded if the analytic group $\operatorname{Inn}_{\mathfrak{g}} \mathfrak{h}$ generated by the set $e^{\text {ad } \mathfrak{h}}$ in Aut $\mathfrak{g}$ has a compact closure. Even for a compactly embedded Cartan algebra $\mathfrak{h}$ of a solvable algebra $\mathfrak{g}$, the analytic group $\operatorname{Inn}_{\mathfrak{g}} \mathfrak{h}$ need not be closed in Aut $_{\mathfrak{g}}$ [HH86]. An element $x \in \mathfrak{g}$ is called compact if $\mathbf{R} \cdot x$ is a compactly embedded subalgebra, and the set of all compact elements of $\mathfrak{g}$ will be denoted comp $\mathfrak{g}$. It is true, although not entirely superficial that a superalgebra is compactly embedded if and only if it is contained in compg.

1. TheOREM (The Uniqueness TheOREM [HH86b]). Let $W$ be an invariant pointed generating cone in a Lie algebra $\mathfrak{g}$. Then

(i) int $W \subseteq$ compg.

(ii) If $H$ is any compactly embedded Cartan algebra, then

(a) $H \cap \operatorname{int} W \neq \varnothing$, and

(b) int $W=\left(\operatorname{Inn}_{\mathfrak{g}} \mathfrak{g}\right) \operatorname{int}_{\mathfrak{h}}(\mathfrak{h} \cap W)$.

In particular, compactly embedded Cartan algebras exist, and if $\mathfrak{h}_{1}$ and $\mathfrak{h}_{2}$ are compactly embedded Cartan algebras and $W_{1}$ and $W_{2}$ are invariant pointed generating cones of $\mathfrak{g}$ such that $\mathfrak{h} \cap W_{1}=\mathfrak{h} \cap W_{2}$, then $W_{1}=W_{2}$.

Received by the editors December 16, 1987.

1980 Mathematics Subject Classification (1985 Revision). Primary 22E60, 22E15. 
This result shows that we know $W$ if we know $\mathfrak{h} \cap W$ for any compactly embedded Cartan algebra $\mathfrak{h}$.

We consider a compactly embedded Cartan algebra $\mathfrak{h}$ and denote by $\Gamma$ the torus $\overline{\operatorname{Inn}_{\mathfrak{g}} \mathfrak{h}}$. Then we obtain the linear projection operator $P: \mathfrak{g} \rightarrow \mathfrak{g}$ by $P(x)=\int_{\Gamma} g(x) d g$ with normalized Haar measure on $\Gamma$. Then $\mathfrak{h}=P(\mathfrak{g})$ and $\mathfrak{g}$ decomposes into a direct sum of $\mathfrak{h}$-modules $\mathfrak{h} \oplus \mathfrak{h}^{+}$with $\mathfrak{h}^{+} \stackrel{\text { def }}{=} \operatorname{ker} P$. For an invariant cone $W$ and any compactly embedded Cartan algebra $\mathfrak{h}$ the meet $\mathfrak{h} \cap W$ and the projection $P(W)$ are related by

$$
P(W)=\mathfrak{h} \cap W .
$$

If $C$ is a pointed cone in a compactly embedded Cartan algebra $\mathfrak{h}$ we define a cone in $\mathfrak{g}$ by

$$
\tilde{C}=\bigcap_{g \in \operatorname{Inn}_{\mathfrak{g}} \mathfrak{g}} g P^{-1}(C)
$$

Then $\tilde{C}=\left\{x \in \mathfrak{g} \mid P\left(\left(\operatorname{Inn}_{\mathfrak{g}} \mathfrak{g}\right) x\right) \subseteq C\right\}$ and $\tilde{C}$ is an invariant cone in $\mathfrak{g}$. Its edge is the largest ideal of $\mathfrak{g}$ contained in $\mathfrak{h}^{+}$. It is not a seriously restrictive assumption that $\mathrm{H}^{+}$should not contain nonzero ideals. Under these circumstances, unfortunately, $\tilde{C}$ may be zero. However, the following theorem uses the device $\tilde{C}$ to reconstruct $W$ from $\mathfrak{h} \cap W$ :

2. THEOREM (THE RECONSTRUCTIONS THEOREM [HH86b]). Suppose that $\mathfrak{h}$ is a compactly embedded Cartan algebra $\mathfrak{h}$ such that $\mathfrak{h}^{+}$contains no nonzero ideal of $\mathfrak{g}$. If $C$ is a pointed generating cone in $\mathfrak{h}$ then the following statements are equivalent:

(A) There exists an invariant pointed cone $W$ in $L$ such that $C=\mathfrak{h} \cap W$.

(B) $C=\mathfrak{h} \cap \tilde{C}$.

(C) Each conjugacy class of an element $c \in C$ projects into $C$ under $P$.

Moreover, if these conditions are satisfied, then $W=\tilde{C}$.

The problem is now to determine which cones $C$ satisfy condition (C) of Theorem 1 and in which Lie algebras they can occur.

3. PROPOSITION [HH86]. Every compactly embedded Cartan subalgebra $\mathfrak{h}$ of $\mathfrak{g}$ is contained in a unique maximal compactly embedded subalgebra $\mathfrak{k}(\mathfrak{h})$. A subalgebra $\mathfrak{k}$ of $\mathfrak{g}$ is maximal compactly embedded if and only if $\operatorname{INN}_{\mathfrak{g}} \mathfrak{k} \stackrel{\text { def }}{=} \overline{\operatorname{Inn}_{\mathfrak{g}} \mathfrak{k}}$ is a maximal compact subgroup of INN $\mathfrak{g}$.

Under the circumstances of Proposition 3, the normalizer $N(\mathfrak{h})$ of the maximal torus $\Gamma=\operatorname{INN}_{g} \mathfrak{h}$ in INN $\mathfrak{g}$ is contained in the compact subgroup $K(\mathfrak{h})=\operatorname{INN}_{\mathfrak{g}} \mathfrak{k}(\mathfrak{h})$. Thus $N(\mathfrak{h}) / \Gamma$ is a finite group, called the Weyl group $\mathscr{W}$ of the pair $(\mathfrak{g}, \mathfrak{h})$. The space $\mathfrak{h}^{+}$is a $\Gamma$-module for the torus $\Gamma$ and thus decomposes into isotypic components. The search for an appropriate natural indexing for such an isotypic component $\mathfrak{v}$ leads to a real linear form $\omega: \mathbf{h} \rightarrow \mathbf{R}$ and a complex structure $I_{\omega}: \mathfrak{h}^{+} \rightarrow \mathfrak{h}^{+}$(that is, a vector space automorphism with $I_{\omega}^{2}=-1$ ) such that the $\mathfrak{h}$-module structure of $\mathfrak{v}$ is given by

$$
[h, x]=\omega(h) \cdot I_{\omega}(x) .
$$


We define

$$
\mathfrak{g}^{\omega}=\left\{x \in \mathfrak{g} \mid\left(\exists I_{\omega}\right) I_{\omega}^{2}=-1 \text { and }(\forall h \in \mathfrak{h})[h, x]=\omega(h) \cdot I x\right\}
$$

We let $\Omega$ denote the set of all $\omega$ for which $\mathfrak{g}^{\omega} \neq\{0\}$ and call these linear forms on $\mathbf{h}$ the real roots of the pair $(\mathfrak{g}, \mathfrak{h})$. We note $\mathfrak{g}^{0}=\mathfrak{h}$. Any choice of a closed half space $E$ in the dual $\hat{\mathfrak{h}}$ of $\mathfrak{h}$ whose boundary hyperplane meets the finite set $\Omega$ only in 0 allows us to represent $\Omega$ as a union $\Omega=\Omega^{+} \cup-\Omega^{+}$with $\Omega^{+}=\Omega \cap E$. We shall call $\Omega^{+}$a selection of positive roots and find the real roots decomposition

$$
\mathfrak{g}=\mathfrak{h} \oplus \mathfrak{h}^{+}, \quad \mathfrak{h}^{+}=\sum_{0 \neq \omega \in \Omega^{+}} \mathfrak{g}^{\omega}
$$

of $\mathfrak{g}$ with respect to $\mathfrak{h}$. The family of complex structures $I_{\omega}$ on $\mathfrak{g}^{\omega}$ then, once a selection of positive roots has been made, gives a complex structure $I$ on $\mathfrak{h}^{+}$with which the bracketing of elements from $\mathfrak{h}$ with those from any $\mathfrak{g}^{\omega}$ is described by

$$
[h, x]=\omega(h) \cdot I x \quad \text { for all } x \in \mathfrak{g}^{\omega} .
$$

At a later point it is important to have available certain special selections of positive roots.

The complex structure $I$ on $\mathfrak{g}^{+}$allows us to define a quadratic function

$$
Q: \mathfrak{h}^{+} \rightarrow \mathfrak{h}, \quad Q(x)=P([I x, x])
$$

For $0 \neq \omega \in \Omega^{+}$and $x \in \mathfrak{g}^{\omega}$ we have

$$
Q(x)=[I x, x]=-[x, I x]
$$

Keep in mind that $Q$ depends on the selection of a set of positive roots via $I$. Changing such a selection may change $Q(x)$ by a sign.

4. PROPOSITION [HH86b, HHL87]. If $\mathfrak{g}$ accommodates an invariant pointed generating cone and $\mathfrak{h}$ is a compactly embedded Cartan algebra, then $Q(x)=0$ and $x \in \mathfrak{g}^{\omega}$ imply $x=0$.

This motivates the following definition.

5. Definition. A Lie algebra $\mathfrak{g}$ is said to have cone potential if it has a compact embedded Cartan algebra $\mathfrak{h}$ and $0 \neq x \in \mathfrak{g}^{\omega}$ for any positive real root $\omega$ implies $Q(x) \neq 0$. 
The structure of Lie algebras with cone potential is special:

6. THEOREM. Let $\mathfrak{g}$ be a Lie algebra with cone potential, $\mathfrak{h}$ a compactly embedded Cartan algebra, $\mathfrak{r}$ its radical, $\mathfrak{n}$ is nilradical, $\mathfrak{z}$ its center. Let $\Omega^{+}$be any selection of positive real roots with respect to $\mathfrak{h}$. For any $\mathfrak{h}$-submodule $\mathbf{v}$ of $\mathfrak{g}$ we write $\mathbf{v}^{\omega}=\mathbf{v} \cap \mathfrak{g}^{\omega}$. Then the following conclusions hold:

(i) $\mathfrak{z}$ is the center of $\mathfrak{n}$ and $\mathfrak{n} / \mathfrak{z}$ is abelian.

(ii)

$$
\left[\mathfrak{n}^{\omega}, \mathfrak{n}^{\omega^{\prime}}\right] \begin{cases}\neq\{0\}, & \text { if } \omega=\omega^{\prime} ; \\ =\{0\}, & \text { if } \omega \neq \omega^{\prime} .\end{cases}
$$

(iii) $\mathfrak{r}^{\omega}=\mathfrak{n}^{\omega}$ for $0 \neq \omega \in \Omega^{+}$.

(iv) There is a Levi complement $\mathfrak{s}$ such that

$$
\mathfrak{h}=(\mathfrak{h} \cap \mathfrak{r}) \oplus(\mathfrak{h} \cap \mathfrak{s}),
$$

and $\mathfrak{h} \cap \mathfrak{s}$ is a compactly embedded Cartan algebra of $\mathfrak{s}$.

(v) $[\mathfrak{h}, \mathfrak{s}] \subseteq \mathfrak{s}$ and $\mathfrak{h}+\mathfrak{s}=(\mathfrak{h} \cap \mathfrak{r}) \oplus \mathfrak{s}$ is a reductive subalgebra.

(vi) $\mathfrak{g}^{\omega}=\mathfrak{r}^{\omega} \oplus \mathfrak{s}^{\omega}$ for $\omega \in \Omega^{+}$.

However, Lie algebras supporting invariant cones are even more special.

7. PROPOSITION [HH86b]. Let $W$ be an invariant pointed generating cone in $\mathfrak{g}$ and let $\mathfrak{h}$ be a compactly embedded Cartan algebra. Then the center $\mathfrak{c}$ of the unique maximal compactly embedded subalgebra $\mathfrak{k}(\mathfrak{h})$ containing $\mathfrak{h}$ contains inner points of comp $\mathfrak{g}$. Moreover, the centralizer of $\mathfrak{c}$ in $\mathfrak{g}$ is $\mathfrak{k}(\mathfrak{h})$.

Such phenomena occur in the context of hermitean symmetric spaces inside semisimple Lie algebras. This motivates the following notation:

8. Definition. A Lie algebra $\mathfrak{g}$ is called quasihermitean if it contains a compactly embedded Cartan algebra $\mathfrak{h}$ such that the center $\mathfrak{c}$ of $\mathfrak{k}(\mathfrak{h})$ satisfies

$$
\mathfrak{c} \cap \operatorname{int}(\operatorname{comp} \mathfrak{g}) \neq \varnothing .
$$

Recalling that $\mathfrak{z}(x)=\operatorname{ker}$ ad $x$ is the centralizer of $x$ in $\mathfrak{g}$, one shows that

$$
\mathfrak{c} \cap \operatorname{int}(\operatorname{comp} \mathfrak{g})=\{x \in \mathfrak{g} \mid \mathfrak{z}(x)=\mathfrak{k}(\mathfrak{h})\}
$$

9. DEFINITION. Let $\Omega$ be the set of real roots of a quaishermitean Lie algebra $\mathfrak{g}$ with respect to a compactly embedded Cartan algebra $\mathfrak{h}$. Then $\omega \in \Omega$ is said to be a compact root if $\mathfrak{g}^{\omega} \subseteq \mathfrak{k}(\mathfrak{h})$. All other roots are noncompact. The set of compact roots is denoted $\Omega_{k}$, the complement is $\Omega_{p}$. For any selection of positive roots $\Omega^{+}$we set $\Omega_{k}^{+}=\Omega^{+} \cap \Omega_{k}$ and $\Omega_{p}^{+}=\Omega^{+} \cap \Omega_{p}$. Finally, we set

$$
\mathfrak{p}(\mathfrak{h})=\bigoplus_{\omega \in \Omega_{p}^{+}} \mathfrak{g}^{\omega}
$$

For any choice of an element $c \in \mathfrak{c} \cap \operatorname{int}(\operatorname{comp} \mathfrak{g})$ there is a selection $\Omega^{+}$of positive roots such that $\omega(c)>0$ for all noncompact roots $\omega$.

10. THEOREM. Let $\mathfrak{g}$ denote a quasihermitean Lie algebra and fix a compactly embedded Cartan algebra $\mathfrak{h}$. Let $\mathfrak{r}$ denote the radical. Then the following 
conclusions hold:

(i) $\mathfrak{k}(\mathfrak{h})=\mathfrak{h} \oplus \bigoplus_{0 \neq \omega \in \Omega_{k}^{+}} \mathfrak{g}^{\omega}$.

(ii) $\mathfrak{g}=\mathfrak{k}(\mathfrak{h}) \oplus \mathfrak{p}(\mathfrak{h})$ and $[\mathfrak{k}(\mathfrak{h}), \mathfrak{p}(\mathfrak{h})] \subseteq \mathfrak{p}(\mathfrak{h})$.

(iii) The unique largest ideal of $\mathfrak{g}$ contained in $\mathfrak{p}(\mathfrak{h})$ contains all ideals $\mathfrak{i}$ with $\mathfrak{h} \cap \mathfrak{i}=\{0\}$.

(iv) $\mathfrak{r} \subseteq \mathfrak{h} \oplus \mathfrak{p}(\mathfrak{h})$.

(v) Let $c \in \mathfrak{c} \cap \operatorname{int}(\operatorname{compg})$ and let $\Omega^{+}$be a selection of positive roots such that $\omega(c)>0$ for all $\omega \in \Omega_{p}^{+}$. Then, with respect to the complex structure $I \mid \mathfrak{p}(\mathfrak{h})$, the vector space $\mathfrak{p}(\mathfrak{h})$ is a complex $k(\mathfrak{h})$-module, i.e., $[k, I p]=I[k, p]$.

It is not hard to record some necessary conditions for a pointed generating cone $C$ in $\mathfrak{h}$ to be of the form $W \cap \mathfrak{h}$. The first is immediate from the definitions

$$
\mathscr{W} C=C \text {. }
$$

A detailed analysis of the orbits of an element $h \in \mathfrak{h}$ under a one-parameter group of inner automorphisms $e^{\mathbf{R} \cdot a d} x$ for a root vector $x \in \mathfrak{g}^{\omega}$ reveals another necessary condition.

For each nonzero real root $\omega \in \Omega$ we define a function $Q_{\omega}: \mathfrak{h} \times \mathfrak{g}^{\omega} \rightarrow \mathfrak{h}$ by $Q_{\omega}(h, x)=\omega(h) \cdot Q(x)=\omega(h) \cdot[I x, x]=\omega(h) \cdot\left[I_{\omega} x, x\right]$. While $I$ and $Q$ depend on a selection of positive roots, the functions $Q_{\omega}$ do not. If $C=\mathfrak{h} \cap W$ for an invariant pointed generating cone $W$, then we find $Q_{\omega}\left(C \times \mathfrak{g}^{\omega}\right) \in C$ for all $\omega \in$ $\Omega_{p}$

This condition is equivalent to

(ROOT) $\quad(\operatorname{ad} x)^{2} C \subseteq C$ for all $x \in L^{\omega}, \omega \in \Omega_{p}$.

The main result is that the two conditions (WEYL) and (ROOT) are also sufficient for $C$ to be of the form $\mathfrak{h} \cap W$.

11. Theorem (The MAIN Characterisation TheOREM). Let $\mathfrak{g}$ denote a quasihermitean Lie algebra with cone potential, and let $\mathfrak{h}$ be a compactly embedded Cartan algebra. Let $C$ be a pointed generating cone in the vector space $\mathfrak{h}$. Then there exists a unique invariant pointed generating cone $W$ in $\mathfrak{g}$ if and only if conditions (WEYL) and (ROOT) are satisfied.

\section{REFERENCES}

[HH85a] J. Hilgert and K. H. Hofmann, Lorentzian cones in real Lie algebras, Monatsh. Math. 100 (1985), 183-210.

[HH85b] —, Old and new on $\mathrm{Sl}(2)$, Manuscripta Math. 54 (1985), 17-52.

[HH85c] _ Lie semialgebras are real phenomena, Math. Ann. 270 (1985), 97-103.

[HH86a] _ , On the automorphism group of cones and wedges, Geom. Dedicata 21 (1986), 205-217.

[HH86b] _ Compactly embedded Cartan algebras and invariant cones in Lie algebras, THD preprint (1986), Adv. in Math. (to appear).

[HHL87] J. Hilgert, K. H. Hofmann and J. D. Lawson, Lie groups, convex cones, and semigroups, Oxford Univ. Press (to appear).

[KR82] S. Kumaresan and A. Ranjan, On invariant convex cones in simple Lie algebras, Proc. Indian Acad. Sci. Math. 91 (1982), 167-182.

[O181] G. I. Ol'shanskiY, Invariant cones in Lie algebras, Lie semigroups and the holomorphic discrete series, Funct. Anal. Appl. 15 (1981), 275-285. 
[Pa84] S. Paneitz, Determination of invariant convex cones in simple Lie algebras, Ark. Mat. 21 (1984), 217-228.

[Vi80] E. B. Vinberg, Invariant cones and orderings in Lie groups, Funct. Anal. Appl. 14 (1980), 1-13.

FACHBEREICH MATHEMATIK, TECHNisChE HOCHSCHUlE DARMSTADT, SCHLOSSGARTENSTR. 7, D-6100 DARMSTADT, FEDERAL REPUBliC OF GERMANY

Electronic address: XMATDA4L @DDATHD21.bitnet 